\title{
Finding community solutions to improve access and acceptance of cataract surgery, optical correction and follow up in children in Malawi
}

\author{
Khumbo Kalua $^{1,2^{*}}$, Francis Masiye ${ }^{3}$, Vincent Jumbe ${ }^{3,4}$, John Barrows $^{5}$, Victoria Sheffield ${ }^{5}$ \\ ${ }^{1}$ Department of Ophthalmology, College of Medicine, University of Malawi, Blantyre, Malawi; \\ *Corresponding Author: khumbokalua@yahoo.com \\ ${ }^{2}$ Blantyre Institute for Community Ophthalmology, Lions Sight First Eye Hospital, Blantyre, Malawi \\ ${ }^{3}$ Department of Community Health, College of Medicine, University of Malawi, Blantyre, Malawi \\ ${ }^{4}$ Centre for Global Health, Trinity College Dublin, Dublin, Ireland \\ ${ }^{5}$ International Eye Foundation, Kensington, Maryland, USA
}

Received 20 January 2013; revised 21 February 2013; accepted 15 March 2013

Copyright (c) 2013 Khumbo Kalua et al. This is an open access article distributed under the Creative Commons Attribution License, which permits unrestricted use, distribution, and reproduction in any medium, provided the original work is properly cited.

\section{ABSTRACT}

Background: Late presentation to the hospital and poor post-operative follow-up after cataract surgery are associated with complications which compromise visual recovery and perpetuate disability among children with cataract. The objectives of the study were to understand the social, psychological and physical consequences of blindness in families, to understand why some parents with blind children access services and others do not, and to explore factors related to decision making within families that prevent access to health care services. Methodology: A mixed methodology quantitative and qualitative community study of blindness in children conducted in southern Malawi to compare "Doers": families with blind children from the same communities who had attended cataract surgical services with "Non-doers" versus families with blind children from the same communities who had not attended services. Individual, family, community socio-cultural and economic characteristics and other qualitative data on knowledge, perceptions, and beliefs were recorded and analyzed thematically, based on grounded theory. Results: A total of 53 in-depth interviews of parents; 21 in-depth interviews of children; 15 focus group discussions with community members; 62 children's clinical eye examinations, and 4 case studies were conducted over the study period. Doer families were likely to have a reliable source of income, have better housing and live closer to health centres than non-doer families. Visual acuity among doers was better than non-doers. Conclusion: This research has highlighted reasons why some families who have children with cataract are likely to be delayed to seek surgical intervention. Comprehensive counseling modules targeting such families need to be developed to increase acceptance and access to children's cataract surgical services.

Keywords: Childhood Blindness; Malawi; Community; Children; Surgery; Optical Correction; Follow up; Blindness

\section{INTRODUCTION}

Cataract (congenital and developmental) is currently the most common cause of reversible blindness and visual impairment in children in developing countries [1,2]. Children can regain their sight and live fully independent productive lives if they are identified early and receive recommended surgical, optical and rehabilitative interventions. If not attended during the critical early period of life [3], amblyopia (lazy eye) may set in resulting in loss of binocular function of vision in the child, and the eye finally develops strabismus [4]. A total of 1.26 million children are blind in the world, with almost a third living in sub-Saharan Africa [5]. It is estimated that there are 100 cataract blind children in every one million child population worldwide. Blindness in children has more than half the number of 'blind years' attributable to cataract in adults [6]. It therefore does not come as a sur- 
prise that the VISION2020 initiative has prioritized blindness in children [7]. The causes of blindness in children are different from adults, implying that different control strategies are needed. A high proportion of children in developing countries suffer from avoidable conditions (preventable and/or treatable) which have well known available treatment modalities (cataract, refractive errors, cornea ulcer, vitamin A deficiency), and are usually non-complicated, non-expensive and often need public health community interventions [8]. New approaches in combating blindness in children need to be deployed in poor countries such as Malawi. The child eye health tertiary facility (CEHTF) [9] in Africa is an approach for combating blindness in children and provides comprehensive pediatric cataract care that includes surgical, optical, and rehabilitative services for a defined catchment area. The Lions Sight First Eye Hospital (LSFEH) located in Blantyre, Malawi is one of the few hospitals qualified under the CEHTF category. However, records over the past four years (2007-2011) indicate that fewer than 100 children per year are admitted for cataract surgery, representing only one third of the total numbers of children expected. Even more worrying is that among those who attend the hospital, up to $50 \%$ present late for surgery, usually one year after the problem has been noted. Among these, a third do not come back for followup after surgery despite assisting the family with resources to defray transportation costs. Both late presentation to the hospital and poor post-operative follow-up are associated with complications which compromise visual recovery and perpetuate disability among children $[10,11]$.

Since 2008, the Blantyre Institute for Community Ophthalmology (BICO), located at the LSFEH, has conducted community research that compares different methods of identifying children from the community in order to ascertain causes of visual impairment and to increase uptake of children presenting with cataract [12,13]. This research used trained volunteers versus health workers and has shown that, despite children being identified in the community and the parents promising that they will bring the children to the hospital, at least a third of such children have not attended the hospital three months after being identified. Proposed reasons for the delays and non-attendance are similar to findings from studies from other countries in Africa [10,11]. These reasons include poor communication among the health providers, families and communities; parents are not aware that treatment for cataract is available; the referral process is complicated and not clearly understood; parents lack the time and money to go to the hospital; and poor (perceived and actual) outcomes of surgery reinforcing fear associated with surgery.

Some of the solutions used by BICO in an attempt to increase the uptake of services for children have involved the Key Informant Method (KIM) [14] and other interventions to overcome barriers such as "lack of knowledge" and "transportation" by providing information and assisting parents and children with transportation refunds. Although these interventions have increased the number of children coming for post-surgery followup examinations, it has not increased the number of children who undergo surgery. These approaches highlight the complexity of reasons for non-attendance suggesting that providing information and transport refunds alone are not sufficient solutions to increase the number of children accepting surgery, and that deeper operational research should be undertaken to identify other deeply rooted reasons in order to create a comprehensive solution.

In this study, we explored some of the factors that may improve blind and visually impaired children's access and acceptance of surgery, optical correction and followup in Malawi.

\section{AIMS AND OBJECTIVES}

The overall objective of the study was to explore some of the factors that may improve blind and visually impaired children access and acceptance to surgery, optical correction and follow up in Malawi.

Specific objectives of the study were to understand the social, psychological and physical consequences of blindness in children and their families; to understand why some families with blind children access services and others do not; to explore factors related to decision making within families that prevent access to health care services and to use the research findings to redesign community interventions to increase uptake of services for blind and visually impaired children. The research compared the clinical social behavior determinants of families from the same geographical areas that accepted surgery (doers) and families that did not receive surgery (non-doers).

\section{METHODS}

\subsection{Ethics Statement}

Ethical approval to conduct the study was obtained from the College of Medicine Research and Ethics Committee (COMREC). The team informed the district health authorities for their permission to conduct the study. The participants gave their written informed consent prior to the interviews. Where the participant was a minor, the guardian (family member) signed consent on their behalf.

The project was undertaken in Zomba, Mangochi and Balaka districts in southern Malawi with a total population of 1.5 million of whom 630,000 are children. A 
community childhood blindness study had been conducted in these districts between 2008 and 2010 and had generated a list of all blind and visually impaired children from the area who were referred and needed to report at the tertiary eye hospital (LSFEH) in Blantyre. The current research utilized this community research database of children, and another two year LSFEH hospital database of approximately 200 children who received cataract surgery during the same period. Children appearing in both databases were enumerated as Doers whereas children appearing in the community database but not the hospital database were enumerated as Non-doers. The research was conducted jointly through collaboration between BICO and the International Eye Foundation (IEF), with involvement of behavioral social scientists from the College of Medicine (VJ \& FM) between January and September 2011.

\subsection{Study Design and Conceptual Framework}

This operational research used a mixed methodology qualitative and quantitative study design. Qualitative data collection methods, focus group discussions and in-depth interviews, were used to collect data from the respondents. The aim was to generate knowledge through a comparison of "Doers"-families with blind children from the same communities who have attended services with "non-doers"-families with blind children from the same communities who had not attended services. Individual family socio-cultural and economic characteristics and other qualitative data on family knowledge, perceptions, and beliefs were recorded and analyzed. The critical behavior examined was whether a family with a child blind from cataract acted on the advice of the health worker and received cataract surgery at the LSFEH. A conceptual framework of behavior change theory was constructed examining internal and external determinants of behavior.

The external behavior determinants, the forces outside of the family and child that affect the behavior, examined were skills and ability to act on the referral advice; barriers to access; cultural beliefs; and consequences of seeking or not seeking treatment. The internal behavior determinants (the forces internal to the family and child that affect how the decision maker thinks and feels about the behavior) were self-efficacy; perceived social norms, perceived risks, perceived consequences and knowledge, and skills that affect decision making process. The questions developed for interviews revolved around three core themes: 1) What are the advantages and disadvantages of accepting, acting on referral advice and receiving the surgery; 2) What would make it easier or more difficult to act upon the referral advice and get the surgery; 3) Who approves and supports or disapproves and inhibits the decision to accept, act on the referral guidelines and undergo surgery.

By including questions that explore external and internal behavioral determinants, the research generated information that was useful in understanding the difficult decisions that families make for their children.

\subsection{Data Collection}

Field work was completed by two teams comprised of two research assistants (RA) and one Ophthalmic Clinical Officer (OCO) who underwent training in qualitative research methods and how to use the research tools. Training was conducted by two senior behavioral social scientists from College of Medicine (VJ \& FM), a member from IEF (JB) and the director of BICO (KK). All data collection tools designed underwent pilot testing. These tools included Focus Group Discussion(FDGs) guides with families of selected Doers/Non-Doers; indepth interview guide for parents of children; in-depth interview guides for selected older children (Doers/NonDoers); eye examination questionnaire of all children using the "WHO" standardized protocol; follow up questionnaire for children who received a follow up clinical investigation more than 2 times after their surgery, and case studies of families who were identified as Doers and Non-Doers.

Interview guides were developed and administered in Chichewa, the local language. All Focus Group Discussions and in-depth interviews took place in a private location. All information obtained in this research was kept strictly confidential to the extent legally possible. Each participant was assigned an identification code, for instance, P1 for Participant \#1, to avoid collecting names. Individual responses were represented by the identification codes. The candidate identification codes were linked to the data generated from focus groups and in-depth interviews. The participants in the focus group discussions and the in-depth interviews were asked to keep the information confidential. A digital voice recorder was used to record conversations and then the recordings were transcribed verbatim into Chichewa and translated into English as soon as possible following the interviews. All data were entered into a computer. Both Chichewa and English transcripts were kept as backup copies both in form of hard paper and electronic copies. Audio recordings were kept for the entire period of the study, in case any material on the transcripts needed to be compared against the original recording. All of the original transcriptions and tapes were stored on a pass-word controlled computer, and the backup copies were locked in the Principal Investigator's filing cabinet. Children's eye examination findings were recorded using an approved WHO form for recording blindness in children.

Ethical approval to conduct the study was obtained 
from the College of Medicine Research and Ethics Committee (COMREC). The team informed the district health authorities for their permission to conduct the study. The participants gave their written informed consent prior to the interviews. Where the participant was a minor, the guardian (family member) signed consent on their behalf. The study excluded newly diagnosed children with visual disabilities.

\subsection{Data Analysis}

Quantitative data were entered in Epidata (version 3.1), exported into excel and STATA and analyzed using STATA SE 10.1 (Stata Corp., Texas, USA). Qualitative data was analyzed thematically, based on grounded theory. The themes were coded by two independent social scientists (VJ \& FM) and the findings were compared. In case of any discrepancies found between the interpretations, the material was reviewed in a meeting and the issue was resolved through agreement. A final report was produced using direct quotations from the participants.

\section{RESULTS}

Table 1 shows the number of planned versus completed interviews, which included in-depth interviews of parents (IDI parents); in-depth interviews with older children $>7$ years (IDI children); focus group discussions with community members (FDG community); child clinical eye examinations; child follow up post surgery examinations, and case studies of affected families.

The interviews (IDI children) were meant to explore how satisfied the children were in regard to the decision taken by the parents on their behalf.

Some of the parents and children could not be tracked and this resulted in fewer interviews than planned. Reasons for not being found were many and included mobile phone contact numbers that had could not be reached; names that could not be traced in the village; and some children reported to have moved away from their original home to another parent/guardian. In terms of FGDs, saturation was reached after conducting 15 interviews. Hence it was felt it was not necessary to complete all of the 18 planned FGDs. Table 2 shows the difference in socioeconomic characteristics between doers and nondoers that may affect their access to eye care services.

There was no significant difference in the mothers education between doers and non-doers $(\mathrm{P}=0.01)$; and there was no significant difference in poverty levels between doers and non-doers using the Malawi poverty (PPI) index $(\mathrm{p}=0.13)$. According to the PPI index, the non-doer score of 24 corresponds to $63.9 \%$ of the families living below the National Poverty Line and $85.6 \%$ living on less than $\$ 1.25$ per day (2005 PPP). In comparison, the doers score of 32 corresponding to $39.4 \%$ of the National Poverty Line and 69\% living on less than $\$ 1.25$ per day (2005 PPP). Even though both doers and non-doers are poor by any standard, the non-doers are much poorer. In terms of distance, non-doers are more likely to live twice as far from the heath centre (first point of contact with the health system) than doers ( $\mathrm{P}=$ 0.01 ). The average distance from health centre to district hospital or tertiary centre does not differ between doers and non-doers.

Non-doers are likely not to have reliable regular sources of income such as employment and are likely to live in poor houses (mud bricks roofed with thatched glasses). Both these factors suggest that non-doers are much poorer than doers.

There were a total of 39 doers of whom 22 (56\%) were boys and a total of 23 non-doers of whom 14 (61\%) were boys. In total $58 \%$ of all children examined were boys and there was no association between gender and whether one was a doer or non-doer $(\mathrm{P}=0.73)$.

Table 3 shows the age group of children between doers and non-doers.

The mean age of children was 9.5 years. There was no association between age groups and whether one was a doer or non-doer $(\mathrm{P}=0.489$, chi 2$)$.

The visual acuity between doers and non-doers in shown in Table 4.

Doers were more likely to have a better presenting visual acuity than non-doers $(P=0.004)$. Non-doers were more likely to have both eyes affected (non-operated) than doers, e.g. presenting with bi-lateral visual impairment (0.033).

Table1. Number of planned and conducted interviews.

\begin{tabular}{cccc}
\hline & No. Planned & No. Conducted & \% Completed \\
\hline IDI: Parents & 73 & 53 & 73 \\
IDI: Children & 27 & 21 & 78 \\
FGD: Community & 18 & 15 & 83 \\
Child: Eye Examination & 77 & 62 & 81 \\
Child: Follow up post surgery & 11 & 11 & 100 \\
Case Studies Families & 4 & 4 & 100 \\
\hline
\end{tabular}


Table 2. Socioeconomic characteristics between families of doers and non-doers.

\begin{tabular}{|c|c|c|c|c|}
\hline & & Doers & Non-doers & P value \\
\hline \multirow[t]{2}{*}{ Education of mother } & Educated $^{*}$ & 23 & 9 & 0.1 \\
\hline & Not educated & 16 & 14 & \\
\hline Overall family poverty & Malawi Poverty Level Index ${ }^{* *}$ & 32 & 24 & 0.13 \\
\hline \multirow[t]{3}{*}{$\begin{array}{l}\text { Distance as an indicator of } \\
\text { access to eye health services }\end{array}$} & Mean distance from village to health center (KM) & 2.4 & 5.5 & 0.01 \\
\hline & Mean distance from health center to district hospital (KM) & 25.8 & 27 & 0.92 \\
\hline & Mean distance from district hospital to tertiary hospital (KM) & 120.8 & 106.8 & 0.5 \\
\hline \multirow[t]{4}{*}{ Sources of income among families } & Peasant farmer & 18 & 15 & \\
\hline & Small scale business & 10 & 4 & \\
\hline & Employed manual labor & 4 & 0 & 0.048 \\
\hline & Employed skilled carpentry, tinsmith etc & 7 & 0 & \\
\hline \multirow[t]{3}{*}{ Housing materials } & Mud bricks with glass thatched & 9 & 15 & \\
\hline & Burnt bricks with glass thatched & 15 & 4 & $<0.001$ \\
\hline & Burnt bricks with metal sheets & 15 & 0 & \\
\hline
\end{tabular}

*Defined as having attended and completed at least the first 5 years of primary education and being able to read and write in the local language; ${ }^{* *}$ The Malawi Poverty Index is derived from the Grameen Foundation Progress out of Poverty Index (PPI) for Malawi. The PPI is a tool for micro-finance programs and consists of ten questions on household size, house construction, water source, cooking fuel, lighting, household ownership of furniture, transportation, and electronics. The scores are converted on a scale of 0 - 100 that are associated with a likely poverty level.

Table 3. Age groups between doers and non-doers.

\begin{tabular}{ccccccc}
\hline & Doer & & Non-doer & & Total & P value \\
\hline & No. & $\%$ & No. & $\%$ & & \\
0 - 5 years & 9 & 23 & 3 & 13 & 12 & \\
6 - 10 years & 15 & 38 & 8 & 35 & 23 & 0.489 \\
11 and above & 15 & 38 & 12 & 52 & 27 & \\
Total & 39 & 100 & 23 & 100 & 62 & \\
\hline
\end{tabular}

\section{Qualitative Findings}

A total of 9 and 6 FGDs were held in doer and nondoer communities, respectively. This resulted in 96 participants being interviewed. Their characteristics are shown in Table 5.

There was no statistically significant difference in gender or education between doers and non-doers.

Table 6 summarizes themes and findings from all IDIs \& FDGs.

An unexpected positive consequence of the interviews was that after the interviews, 11 out of 23 (48\%) "Nondoer" parents converted to doers (accepted surgery and came with their children to the hospital immediately after the interviews).

\section{DISCUSSION}

This operational research study was designed to explore reasons why some parents with blind children access services and others do not, and, to explore factors in the family related to decision making that prevent theca- cess of health care services. In rural areas of Malawi, there are challenges in tracing families of affected children in the community. One challenge is that persons within a village are known by different names, and the name recorded on the database though official, may not be a name fellow villagers recognize. It is therefore not surprising that some IDI's could not be conducted with parents as they could not be identified at that particular time in the mentioned village.

There are important, but minor differences identified between the doers and the non-doers. The non-doer families are poorer, live further from the nearest health center, and their children are more likely to have cataract in both eyes than the children of doer families. Although education of the mother in this study was not associated with educated mothers being doers, several studies have shown that an educated mother is more likely to seek a health intervention for her child than a non-educated mother $[10,11,15]$. It is possible that this effect could not be shown in this study, but because the study sample size was small, the study might not have had enough power to show the difference. Poverty and a lack of education make it difficult for any family to access health services, as these families may have less frequent contact with the health services (due to poverty). Even if they attended health services, their lack of education may hinder their ability to maximally benefit from health communication messages.

Distance has always been known as a barrier to accessing health services. However this study has shown that in Malawi it is not the distance from health centre to the district hospital or indeed to tertiary hospital, but 
Table 4. Visual acuity between doers and non-doers.

\begin{tabular}{|c|c|c|c|c|c|c|}
\hline & \multicolumn{2}{|l|}{ Doer } & \multicolumn{2}{|c|}{ Jon-doer } & \multirow[b]{2}{*}{ Total } & \multirow[b]{2}{*}{$\%$} \\
\hline & No. & $\%$ & No. & $\%$ & & \\
\hline Normal & 26 & 67 & 7 & 30 & 33 & 31 \\
\hline Visual impaired & 6 & 15 & 5 & 22 & 11 & 32 \\
\hline Severely visually impaired & 4 & 10 & 1 & 4 & 5 & 8 \\
\hline Blind* & 3 & 8 & 10 & 43 & 13 & 21 \\
\hline Total & 39 & 100 & 23 & 100 & 62 & 100 \\
\hline P value $0.004^{*}$ & & & & & & \\
\hline
\end{tabular}

Table 5. Characteristics of participants in FDGs.

\begin{tabular}{cccc}
\hline & & No. & $\%$ \\
\hline Gender & Male & 44 & 46 \\
& Female & 52 & 54 \\
Education level & None & 21 & 22 \\
& Primary & 47 & 49 \\
& Secondary & 28 & 29 \\
Total & & 96 & $100 \%$ \\
\hline
\end{tabular}

rather it is the distance from the village to the nearest health centre that matters more. Doers are likely to be twice as nearer to the health centre than non-doers. It is possible that being closer to the health centre exposes doers to more health promotion messages at the health centre, and enables doers to form a closer personal relationship with health centre staff. These factors may be associated with increased access to the health care. Finally, often the most common means of accessing a health centre from a village is by walking, and those far away are likely to be reluctant to walk, especially when the eye condition is not painful or life threatening.

Although the socio-economic indicators identified are important, the other key but less tangible determinants that influence behavior are equally important as they provide a greater understanding of what individual families and community members believe about blindness and establish factors that influence family decisions to seek care. While it is difficult to summarize these differences, it is useful to understand some of these in the context in which families make decisions. Communities have limited knowledge and understanding of cataract in children and its management. Cataract is frequently confused with corneal opacity. This should not be surprising because these communities have been familiar with the problems of vitamin A deficiency and xerophthalmia in the recent past indicating their understanding that blinding malnutrition can cause blindness in children. It was clear from the interviews that even though families understand that blindness is a burden, they do not equate cataract with blindness as they have some idea that once one has a cataract they can be operated any time later and can see again. This disassociation may hinder families with children attending services earlier, and delays could lead to poor visual recovery. Encouraging a broader family decision making process while at the same time addressing demotivating factors, is likely to lead to more acceptance of surgery.

The unintended consequence of non-doers becoming doers after the interview intervention is a direct result of more time being spent in talking to the families and communities and suggests that comprehensive counseling is needed. Finally, the visual acuity obtained from clinical examinations clearly shows that a good number of children who undergo surgery (doers) end up with acceptable levels of visual acuity. This information together with information obtained from qualitative findings can be used to develop counseling tools that can increase uptake of surgery and follow up in children with cataract.

\section{CONCLUSION}

In conclusion, this operational research has highlighted the complexities involved in understanding behavior and finding solutions to increase acceptance and access to surgical services by children. A counseling module can be developed based on the interview questions, can be tested and modified at a low cost, and can make part of the Key Informant Method.

\section{ACKNOWLEDGEMENTS}

We sincerely thank the following Research Assistants for their hard work and enthusiastic contribution to this study during data collection and data processing: Misheck Nyirenda, Deborah Mwafulirwa, and Christopher Ndembo. We also thank the clinicians, Bruno Chimaliro and George Moyo who accompanied the Research Assistants in the field and conducted eye examinations. 
Table 6. Main themes from qualitative interviews.

\begin{tabular}{|c|c|}
\hline Main theme & Summary of qualitative findings between doers and non doers \\
\hline & "It is an opacity at the centre of the eye" \\
\hline Local language understanding of cataract & $\begin{array}{l}\text { Interpretation: Both groups understand what a cataract is : but the same local terminology } \\
\text { applies for a cornea scar }\end{array}$ \\
\hline & “one gets an eye infection and when that heals the white cataract develops) \\
\hline causes and symptoms of cataract & Interpretation: Often confused with other conditions such as cornea scarring \\
\hline Linkage between a cataract and blindness & "Blindness is when a child can only see dark; with cataract a child sees light" \\
\hline Linkage vetween a cataract and vimuness & Interpretation: difference not clearly understood. \\
\hline & Interpretation: difference not clearly understood. \\
\hline \multirow{4}{*}{ Perceived consequences of untreated child cataract } & "a child will have be in trouble the rest of his life if he cannot see" \\
\hline & Interpretation: Both groups perceive having cataract is not good for a child. \\
\hline & "My husband’s family advised us to take a child to a hospital” Doer \\
\hline & "The father does not want to listen; he has refused to take the child to the hospital" Non \\
\hline \multirow[t]{2}{*}{ Health seeking decisions } & Doer \\
\hline & $\begin{array}{l}\text { Interpretation: Doers often had multiple family members involved; non doers were } \\
\text { individuals families who did not consult other family members }\end{array}$ \\
\hline \multirow{2}{*}{ Demotivating factors } & Interpretation: Fear of surgery for both doers and non doers: \\
\hline & "If he goes to school, he can be like anyone else” Doer \\
\hline \multirow{2}{*}{ Future of a blind child } & “He will never learn, it’s a helpless situation” \\
\hline & $\begin{array}{l}\text { Interpretation: Doers believe blind children can perform and achieve same as sighted if } \\
\text { given a chance: non doers believe blind child has no future and situation is helpless }\end{array}$ \\
\hline
\end{tabular}

This research was supported by the International Eye Foundation under the "A2Z Child Blindness and Health Grants Fund" supported by the US Agency for International Development (SUBAGREEMENT No. 3567-IEF-00, Under USAID Cooperative Agreement Number GHS-A00-05-00012-00.

\section{AUTHORS CONTRIBUTION}

Conceived and designed the experiments: KK JB.

Performed the experiments: KK .

Analyzed the data: KK VM FM.

Contributed reagents/materials/analysis tools: KK JB.

Wrote the paper: KK VS VM FM.

\section{REFERENCES}

[1] Gilbert, C. and Rahi, J. (2010) Visual impairment and blindness in children. In: Johnson, G., Foster, A. and Gilbert, C., Eds., Epidemiology of Eye Disease, 3rd Edition, Imperical College Press, London.

[2] Muhit, M.A., Shah, S.P., Gilbert, C.E. and Foster, A. (2007) Causes of severe visual impairment and blindness in Bangladesh: A study of 1935 children. British Journal of Ophthalmology, 91, 1000-1004. http://dx.doi.org/10.1136/bjo.2006.108019

[3] Day, S. (1997) Normal and abnormal visual development. In: Taylor, D., Ed., Paediatric Ophthalmology, 2nd Edition, Blackwell Science, London, 13-28.

[4] Von Noorden, G.K. (1980) Binocular vision and ocular motility. CV Mosby, St. Louis.

[5] Chandna, A. and Gilbert, C. (2010) When your eye patient is a child. Community Eye Health, 23, 1-3.

[6] Gilbert, C., Rahi, J. and Quinn, E. (2003) Visual impairment and blindness in children. In: Johnson, G., Minassian, D., Weale, R. and West, S., Eds., Epidemiology of
Eye Disease, 3rd Edition, Arnold, London, 260-285.

[7] Gilbert, C. and Foster, A. (2001) Childhood blindness in the context of VISION 2020-The right to sight. Bulletin of the World Health Organization, 79, 227-232.

[8] Rahi, J.S. and Cable, N. (2003) Severe visual impairment and blindness in children in the UK. Lancet, 362, 13591365. http://dx.doi.org/10.1016/S0140-6736(03)14631-4

[9] Agarwal, P.K., Bowman, R. and Courtright, P. (2010) Child eye health tertiary facilities in Africa. Journal of AAPOS, 14, 263-266. http://dx.doi.org/10.1016/j.jaapos.2010.02.007

[10] Bronsard, A., Geneau, R., Shirima, S., Courtright, P. and Mwende, J. (2008) Why are children brought late for cataract surgery? Qualitative findings from Tanzania. Ophthalmic Epidemiology, 15, 383-388. http://dx.doi.org/10.1080/09286580802488624

[11] Mwende, J., Bronsard, A., Mosha, M., Bowman, R., Geneau, R., et al. (2005) Delay in presentation to hospital for surgery for congenital and developmental cataract in Tanzania. British Journal of Ophthalmology, 89, 14781482. http://dx.doi.org/10.1136/bjo.2005.074146

[12] Kalua, K., Patel, D., Muhit, M. and Courtright, P. (2008) Causes of blindness among children identified through village key informants in Malawi. Canadian Journal of Ophthalmology, 43, 425-427. http://dx.doi.org/10.3129/I08-084

[13] Kalua, K., Patel, D., Muhit, M. and Courtright, P. (2009) Productivity of key informants for identifying blind children: Evidence from a pilot study in Malawi. Eye (London), 23, 7-9. http://dx.doi.org/10.1038/eye.2008.49

[14] Muhit, M.A., Shah, S.P., Gilbert, C.E., Hartley, S.D. and Foster, A. (2007) The key informant method: A novel means of ascertaining blind children in Bangladesh. British Journal of Ophthalmology, 91, 995-999. http://dx.doi.org/10.1136/bjo.2006.108027

[15] Semba, R.D., de Pee, S., Sun, K., Akhter, N., Bloem, 
M.W., et al. (2010) Coverage of vitamin A capsule programme in Bangladesh and risk factors associated with non-receipt of vitamin A. Journal of Health, Population and Nutrition, 28, 143-148.

http://dx.doi.org/10.3329/jhpn.v28i2.4884 